Pacific

Journal of

Mathematics

\title{
DEGREE-THREE SPIN HURWITZ NUMBERS
}

JUNHO LEE

Volume 263 No. 2

June 2013 


\title{
DEGREE-THREE SPIN HURWITZ NUMBERS
}

\author{
JUNHO LEE
}

\section{Gunningham (2012) calculated all spin Hurwitz numbers in terms of com- binatorics of the Sergeev algebra. Here we use a spin curve degeneration to obtain a recursion formula for degree-three spin Hurwitz numbers.}

Let $D$ be a complex curve of genus $h$ and $N$ be a theta characteristic on $D$, that is, $N^{2}=K_{D}$. The pair $(D, N)$ is called a spin curve of genus $h$ with parity $p \equiv h^{0}(N)(\bmod 2)$. For $i=1, \ldots, k$, let $m^{i}=\left(m_{1}^{i}, \ldots, m_{\ell_{i}}^{i}\right)$ be an odd partition of $d>0$, namely, all components $m_{j}^{i}$ are odd. Fix $k$ points $q^{1}, \ldots, q^{k}$ in $D$ and consider degree- $d$ maps $f: C \rightarrow D$ from possibly disconnected domains $C$ of Euler characteristic $\chi$ that are ramified only over the fixed points $q^{i}$ with ramification data $m^{i}$. Observe that the Riemann-Hurwitz formula shows

$$
2 d(1-h)-\chi+\sum_{i=1}^{k}\left(\ell\left(m^{i}\right)-d\right)=0,
$$

where $\ell\left(m^{i}\right)=\ell_{i}$ is the length of $m^{i}$. By the Hurwitz formula, the twisted line bundle

$$
L_{f}=f^{*} N \otimes \mathcal{O}\left(\sum_{i, j} \frac{1}{2}\left(m_{j}^{i}-1\right) x_{j}^{i}\right)
$$

is a theta characteristic on $C$ where $f^{-1}\left(q^{i}\right)=\left\{x_{j}^{i}\right\}_{1 \leq j \leq \ell_{i}}$ and $f$ has multiplicity $m_{j}^{i}$ at $x_{j}^{i}$. We define the parity $p(f)$ of a map $f$ by

$$
p(f) \equiv h^{0}\left(L_{f}\right)(\bmod 2) .
$$

Given odd partitions $m^{1}, \ldots, m^{k}$ of $d$, the spin Hurwitz number of genus $h$ and parity $p$ is defined as a (weighted) sum of (ramified) covers $f$ satisfying (0-1) with sign determined by the parity $p(f)$ :

$$
H_{m^{1}, \ldots, m^{k}}^{h, p}=\sum_{f} \frac{(-1)^{p(f)}}{|\operatorname{Aut}(f)|}
$$

MSC2010: 14N35, 53D45.

Keywords: local Gromov-Witten invariants, spin curve, Schiffer variation. 
Eskin, Okounkov, and Pandharipande [Eskin et al. 2008] calculated the genus $h=1$ and odd parity spin Hurwitz numbers in terms of characters of the Sergeev group. Gunningham [2012] calculated all spin Hurwitz numbers in terms of combinatorics of the Sergeev algebra.

The trivial partition $\left(1^{d}\right)$ of $d$ is a partition whose components are all 1 . If $m^{k}=\left(1^{d}\right), f$ has no ramification points over the fixed point $q^{k}$ and hence we have

$$
H_{m^{1}, \ldots, m^{k-1},\left(1^{d}\right)}^{h, p}=H_{m^{1}, \ldots, m^{k-1}}^{h, p} .
$$

When all partitions $m^{i}=\left(1^{d}\right)$, denote the spin Hurwitz numbers (0-4) by $H_{d}^{h, p}$. These are dimension-zero local GW invariants $G T_{d}^{\text {loc, } h, p}$ of spin curve $(D, N)$ that give all dimension-zero GW invariants of Kähler surfaces with a smooth canonical divisor; see [Kiem and Li 2007; 2011; Lee and Parker 2007; Maulik and Pandharipande 2008]. For notational simplicity, we set $H_{(3)^{0}}^{h, p}=H_{3}^{h, p}$ and for $k \geq 1$ write

$$
H_{(3)^{k}}^{h, p}
$$

for the spin Hurwitz numbers $H_{(3), \ldots,(3)}^{h, p}$ with the same $k$ partitions (3). Since there are two odd partitions $\left(1^{3}\right)$ and (3) of $d=3$, by (0-5) it suffices to compute $H_{(3)^{k}}^{h, p}$ for $k \geq 0$. The aim of this paper is to use a spin curve degeneration to obtain the following recursion formula.

Theorem 0.1. If $h=h_{1}+h_{2}$ and $p \equiv p_{1}+p_{2}(\bmod 2)$, then, for $k_{1}+k_{2}=k$,

$$
H_{(3)^{k}}^{h, p}=3 ! H_{(3)^{k_{1}}}^{h_{1}, p_{1}} \cdot H_{(3)^{k_{2}}}^{h_{2}, p_{2}}+3 H_{(3)^{k_{1}+1}}^{h_{1}, p_{1}} \cdot H_{(3)^{k_{2}+1}}^{h_{2}, p_{2}} .
$$

One can use Theorem 0.1 and the result of [Eskin et al. 2008] to explicitly compute the spin Hurwitz numbers of degree $d=3$. In Proposition 7.1, we show that

$$
H_{(3)^{k}}^{h, \pm}=3^{2 h-2}\left[(-1)^{k} 2^{k+h-1} \pm 1\right],
$$

where + and - denote the even and odd parities. When the degree $d$ is 1 or 2 , the dimension-zero local GW invariants are given by the formulas

$$
G T_{1}^{\mathrm{loc}, h, \pm}= \pm 1 \quad \text { and } \quad G T_{2}^{\mathrm{loc}, h, \pm}= \pm 2^{h-1}
$$

see Lemma 2.6 of [Lee 2013]. Since $G T_{d}^{\text {loc, } h, p}=H_{d}^{h, p}$ as mentioned above, formula (0-7) shows

$$
G T_{3}^{\mathrm{loc}, h, \pm}=3^{2 h-2}\left(2^{h-1} \pm 1\right) .
$$

This calculation is, in fact, the main motivation for the paper.

In Section 1, we express the degree- $d$ spin Hurwitz numbers (0-4) in terms of relative GW moduli spaces. We can then apply a degeneration method for a family of curves $\mathscr{D} \rightarrow \Delta$ where the central fiber $D_{0}$ is a nodal curve and the general fiber 
$D_{\lambda}(\lambda \neq 0)$ is a smooth curve. Section 2 describes the relative moduli space $\mu_{0}$ of maps $f$ into the nodal curve $D_{0}$. In Section 3, we show that the union over $\lambda \in \Delta$ of relative moduli spaces $M_{\lambda}$ of maps into $D_{\lambda}$ consists of connected components $\mathscr{E}_{m, f} \rightarrow \Delta$ containing $f \in M_{0}$. Here $m$ is the ramification data of $f$ over nodes of $D_{0}$ such that $d-\ell(m)$ is even.

The (ordinary) Hurwitz numbers are sums of (ramified) maps modulo automorphism without sign. One can easily obtain a recursion formula for Hurwitz numbers by counting maps in the general fiber of $\mathscr{L}_{m, f} \rightarrow \Delta$. For spin Hurwitz numbers, one needs to calculate parities of maps induced from a fixed spin structure on the family of curves $\mathscr{D}$.

The novelty of our approach is to apply a Schiffer variation for the parity calculation. The space $\mathscr{L}_{m, f}$ is, in general, not smooth. In Section 4, we construct a smooth model for $\mathscr{L}_{m, f}$ by Schiffer variation. In Section 5, we use the smooth model to twist the pullback of the spin structure on $\mathscr{D}$. When the degree $d$ equals 3 , the partition $m$ is odd, either $\left(1^{3}\right)$ or (3). In this case, a suitable twisting immediately yields a required parity calculation. We prove Theorem 0.1 in Section 6 and formula (0-7) in Section 7.

For higher degree $d \geq 4$, the partition $m$ may not be odd! A new parity calculation is needed. In [Lee and Parker 2012], we generalized the recursion formula (0-6) for higher-degree spin Hurwitz numbers by employing additional geometric analysis arguments for parity calculations.

\section{Dimension zero relative GW moduli spaces}

In this section, we express the spin Hurwitz numbers (0-4) in terms of dimensionzero relative GW moduli spaces. We follow the definitions of [Ionel and Parker 2003] for the relative GW theory.

Let $D$ be a smooth curve of genus $h$ and let $V=\left\{q^{1}, \ldots, q^{k}\right\}$ be a fixed set of points on $D$. Given partitions $m^{1}, \ldots, m^{k}$ of $d$, a degree- $d$ holomorphic map $f: C \rightarrow D$ from a possibly disconnected curve $C$ is called $V$-regular with contact vectors $m^{1}, \ldots, m^{k}$ if $f^{-1}(V)$ consists of $\sum \ell\left(m^{i}\right)$ contact marked points $x_{j}^{i}(1 \leq$ $\left.j \leq \ell\left(m^{i}\right)\right)$ with $f\left(x_{j}^{i}\right)=q^{i}$ such that $f$ has ramification index (or multiplicity) $m_{j}^{i}$ at $x_{j}^{i}$. Two $V$-regular maps $\left(f, C ;\left\{x_{j}^{i}\right\}\right)$ and $\left(\tilde{f}, \widetilde{C} ;\left\{\tilde{x}_{j}^{i}\right\}\right)$ are equivalent if they are isomorphic, that is, there is a biholomorphism $\sigma: C \rightarrow \widetilde{C}$ with $\tilde{f} \circ \sigma=f$ and $\sigma\left(x_{j}^{i}\right)=\tilde{x}_{j}^{i}$ for all $i, j$. The relative moduli space

$$
M_{\chi, m^{1}, \ldots, m^{k}}^{V}(D, d)
$$

consists of equivalence classes of $V$-regular maps $\left(f, C ;\left\{x_{j}^{i}\right\}\right)$ with the Euler characteristic $\chi(C)=\chi$ and with contact vectors $m^{1}, \ldots, m^{k}$. Since no confusion can 
arise, we regard a point in the space (1-1) as a $V$-regular map $\left(f, C ;\left\{x_{j}^{i}\right\}\right)$. For simplicity, we often write a $V$-regular map $\left(f, C ;\left\{x_{j}^{i}\right\}\right)$ simply as $f$.

The (formal) complex dimension of the space (1-1) is given by the left side of the Riemann-Hurwitz formula (0-1):

$$
2 d(1-h)-\chi-\sum_{i=1}^{k}\left(d-\ell\left(m^{i}\right)\right) .
$$

Suppose this dimension is zero. Then, for each $V$-regular map $\left(f, C ;\left\{x_{j}^{i}\right\}\right)$ in (1-1), forgetting the contact marked points $x_{j}^{i}$ gives a (ramified) cover $f$ that is ramified only over fixed points $q^{i}$ and satisfies (0-1). The automorphism group $\operatorname{Aut}(f)$ of a (ramified) cover $f$ consists of automorphisms $\sigma \in \operatorname{Aut}(C)$ with $f \circ \sigma=f$. The automorphism group $\operatorname{Aut}(f, V)$ of a $V$-regular map $\left(f, C ;\left\{x_{j}^{i}\right\}\right)$ consists of automorphisms $\sigma \in \operatorname{Aut}(f)$ with $\sigma\left(x_{j}^{i}\right)=x_{j}^{i}$ for all $i, j$.

For a partition $m$ of $d$, let Aut $(m)$ be the subgroup of symmetric group $S_{\ell(m)}$ permuting equal parts of the partition $m$.

Lemma 1.1. Let $m^{1}, \ldots, m^{k}$ be as above and suppose the dimension (1-2) is zero.

(a) If $m^{i}=\left(1^{d}\right)$ for some $1 \leq i \leq k, \operatorname{Aut}(f, V)$ is trivial for all $f$ in (1-1).

(b) If $m^{1}, \ldots, m^{k}$ are all odd partitions,

$$
H_{m^{1}, \ldots, m^{k}}^{h, p}=\frac{1}{\prod_{i=1}^{k}\left|\operatorname{Aut}\left(m^{i}\right)\right|} \sum \frac{(-1)^{p(f)}}{|\operatorname{Aut}(f, V)|}
$$

where the sum is over all $f$ in (1-1) and $p(f)$ is the parity (0-3).

Proof. Let $\left(f, C ;\left\{x_{j}^{i}\right\}\right)$ be a $V$-regular map in (1-1) and $\sigma \in \operatorname{Aut}(f, V)$. If $m^{i}=\left(1^{d}\right)$, the set of branch points $B$ of $f$ is a subset of $V \backslash\left\{q^{i}\right\}$ and the restriction of $\sigma$ to $C \backslash f^{-1}(B)$ is a covering transformation that fixes contact marked points $x_{1}^{i}, \ldots, x_{d}^{i}$. Noting $f^{-1}(B)$ is finite, we conclude that $\sigma$ is an identity map on $C$. This proves (a).

As mentioned above, forgetting contact marked points $x_{j}^{i}$ gives a (ramified) cover $f$ satisfying (0-1). Conversely, given a (ramified) cover $f$ satisfying (0-1), one can mark a point over $q^{i}$ with ramification index $m_{j}^{i}$ as a contact marked point $x_{j}^{i}$. Such marking gives $V$-regular maps $\left(f, C ;\left\{x_{j}^{i}\right\}\right)$ in $\prod_{i=1}^{k}\left|\operatorname{Aut}\left(m^{i}\right)\right|$ ways. Observe that $\left(f, C ;\left\{x_{j}^{i}\right\}\right)$ and $\left(f, C ;\left\{\sigma\left(x_{j}^{i}\right)\right\}\right)$ are isomorphic for each $\sigma \in \operatorname{Aut}(f)$ and that $\operatorname{Aut}(f, V)$ is a normal subgroup of $\operatorname{Aut}(f)$. Consequently, the quotient group $G=\operatorname{Aut}(f) / \operatorname{Aut}(f, V)$ acts freely on the set of $V$-regular maps $\left(f, C ;\left\{x_{j}^{i}\right\}\right)$ obtained by the (ramified) cover $f$. Its orbits give $\prod_{i=1}^{k}\left|\operatorname{Aut}\left(m^{i}\right)\right| /|G|$ points (that is, equivalence classes of $V$-regular maps) in the space (1-1), each of which has the same automorphism group $\operatorname{Aut}(f, V)$. Now (b) follows from counting maps with the parity of map modulo automorphisms. 


\section{Maps into a nodal curve}

Let $D_{0}=D_{1} \cup E \cup D_{2}$ be a connected nodal curve of (arithmetic) genus $h$ with two nodes $p^{1}$ and $p^{2}$ such that, for $i=1,2, E=\mathbb{P}^{1}$ meets $D_{i}$ at node $p^{i}$ and $D_{i}$ has genus $h_{i}$ with $h_{1}+h_{2}=h$. In this section, we consider maps into $D_{0}$ that are relevant to our subsequent discussion.

Below, we fix $d, h, \chi$, and odd partitions $m^{1}, \ldots, m^{k}$ of $d$ so that the RiemannHurwitz formula (0-1) holds, or equivalently, the dimension formula (1-2) is zero. For each partition $m$ of $d$, consider the product space

$\mathscr{P}_{m}=\mathcal{M}_{\chi_{1},\left(1^{d}\right), m^{1}, \ldots, m^{k_{1}, m}}^{V_{1}}\left(D_{1}, d\right) \times \mathcal{M}_{\chi_{0}, m,\left(1^{d}\right), m}^{V_{0}}(E, d) \times \mathcal{M}_{\chi_{2}, m, m^{k_{1}+1}, \ldots, m^{k},\left(1^{d}\right)}^{V_{2}}\left(D_{2}, d\right)$

where

$V_{1}=\left\{q^{k+1}, q^{1}, \ldots, q^{k_{1}}, p^{1}\right\}, \quad V_{0}=\left\{p^{1}, q^{k+2}, p^{2}\right\}, \quad V_{2}=\left\{p^{2}, q^{k_{1}+1}, \ldots, q^{k}, q^{k+3}\right\}$

and

$$
\chi_{1}+\chi_{0}+\chi_{2}-4 \ell(m)=\chi .
$$

For simplicity, let $\mathcal{M}_{m}^{1}, \mathcal{M}_{m}^{0}$, and $\mathcal{M}_{m}^{2}$ denote the first, second, and third factors of $\mathscr{P}_{m}$.

Lemma 2.1. If $\mathscr{P}_{m} \neq \varnothing$, the spaces $\mathcal{M}_{m}^{1}, \mathcal{M}_{m}^{0}$, and $\mathcal{M}_{m}^{2}$ have dimension zero. Consequently, $\chi_{0}=2 \ell(m)$ and $d-\ell(m)$ is even.

Proof. Each $\mathcal{M}_{m}^{i}(0 \leq i \leq 2)$ has nonnegative dimension by the Riemann-Hurwitz formula. The formula (2-1) and our assumption that the dimension (1-2) is zero thus imply that each $M_{m}^{i}$ has dimension zero. The dimension formulas for $M_{m}^{0}$ and $M_{m}^{i}(i=1,2)$ then show that $\chi_{0}=2 \ell(m)$ and $d-\ell(m)$ is even because $d-\ell\left(m^{i}\right)=\sum\left(m_{j}^{i}-1\right)$ is even for all $1 \leq i \leq k$.

Let $|A|$ denote the cardinality of a set $A$.

\section{Lemma 2.2.}

$$
\left|\mathcal{M}_{m}^{0}\right|=\frac{d !|\operatorname{Aut}(m)|}{\prod m_{j}} .
$$

Proof. Let $f \in M_{m}^{0}$. Since $\chi_{0}=2 \ell(m)$, the domain of $f$ is a disjoint union of smooth rational curves $E_{j}$ for $1 \leq j \leq \ell(m)$, and each restriction $f_{j}=\left.f\right|_{E_{j}}$ has exactly one contact marked point over $p^{i}(i=1,2)$ with multiplicity $m_{j}$, so $f_{j}$ has degree $m_{j}$.

Consequently, forgetting contact marked points of maps in $\mu_{m}^{0}$ gives exactly one map (as a cover) with automorphism group of order $|\operatorname{Aut}(m)| \prod m_{j}$. Here the factor $|\operatorname{Aut}(m)|$ appears because we can relabel maps $f_{j}$ in $|\operatorname{Aut}(m)|$ ways and the factor $\prod m_{j}$ appears because each restriction map $f_{j}$ (as a cover) has an automorphism group of order $m_{j}$. We then argue as in the proof of Lemma 1.1. 
For each $\left(f_{1}, f_{0}, f_{2}\right) \in \mathscr{P}_{m}$, by identifying contact marked points over $p^{i} \in D^{i} \cap E$ $(i=1,2)$, one can glue the domains of $f_{i}$ and $f_{0}$ to obtain a map $f: C \rightarrow D_{0}$ with $\chi(C)=\chi$. For notational convenience, we often write the glued map $f$ as $f=\left(f_{1}, f_{0}, f_{2}\right)$. Denote by

$$
M_{m, 0}
$$

the space of such glued maps $f=\left(f_{1}, f_{0}, f_{2}\right)$. Contact marked points are labeled, but nodal points of $C$ are not labeled. Thus, we have the following.

Lemma 2.3. $\mathscr{P}_{m}$ is a cover of $\mathcal{M}_{m, 0}$ of degree $|\operatorname{Aut}(m)|^{2}$.

\section{Limiting and gluing}

Following [Ionel and Parker 2004], this section describes limiting and gluing arguments under a degeneration of target curves. Let $D_{0}=D_{1} \cup E \cup D_{2}$ be the nodal curve with fixed points $q^{1}, \ldots, q^{k+3}$ as in Section 2. In Section 4, we construct a family of curves together with $k+3$ sections:

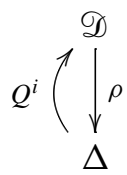

Here the total space $\mathscr{D}$ is a smooth complex surface, $\Delta \subset \mathbb{C}$ is a disk with parameter $\lambda$, the central fiber is $D_{0}$, the general fiber $D_{\lambda}(\lambda \neq 0)$ is a smooth curve of genus $h$, and $Q^{i}(0)=q^{i}$ for $1 \leq i \leq k+3$. By Gromov's convergence theorem, a sequence of holomorphic maps into $D_{\lambda}$ with $\lambda \rightarrow 0$ has a map into $D_{0}$ as a limit. For notational simplicity, for $\lambda \neq 0$ we set

$$
\mu_{\lambda}=M_{\chi, m^{1}, \ldots, m^{k+3}}^{V_{\lambda}}\left(D_{\lambda}, d\right), \quad \text { where } V_{\lambda}=\left\{Q^{1}(\lambda), \ldots, Q^{k+3}(\lambda)\right\},
$$

and denote the set of limits of sequences of maps in $M_{\lambda}$ as $\lambda \rightarrow 0$ by

$$
\lim _{\lambda \rightarrow 0} M_{\lambda} \text {. }
$$

Lemma 3.1 shows that limit maps in (3-3) lie in the union of spaces (2-2), namely,

$$
\lim _{\lambda \rightarrow 0} M_{\lambda} \subset \bigcup_{m} M_{m, 0}
$$

where the union is over all partitions $m$ of $d$ with $d-\ell(m)$ even.

Conversely, by the gluing theorem of [Ionel and Parker 2004], the domain of each map in $\mathcal{M}_{m, 0}$ can be smoothed to produce maps in $\mathcal{M}_{\lambda}$ for small $|\lambda|$. Shrinking $\Delta$ if necessary, for $\lambda \in \Delta$, one can assign to each $f_{\lambda} \in \mathcal{M}_{\lambda}$ a partition $m$ of $d$ by (3-4). Let $M_{m, \lambda}$ be the set of all pairs $\left(f_{\lambda}, m\right)$. For each $f \in \mathcal{M}_{m, 0}$, let

$$
\mathscr{L}_{m, f} \rightarrow \Delta
$$


be the connected component of $\bigcup_{\lambda \in \Delta} M_{m, \lambda} \rightarrow \Delta$ that contains $f$, and let

$$
\mathscr{Z}_{m, f, \lambda}
$$

denote the fiber of (3-5) over $\lambda \in \Delta$. It follows that, for $\lambda \neq 0$,

$$
M_{\lambda}=\bigsqcup_{f \in M_{m, 0}} \mathscr{E}_{m, f, \lambda} .
$$

For $f=\left(f_{1}, f_{0}, f_{2}\right) \in M_{m, 0}$ where $m=\left(m_{1}, \ldots, m_{\ell}\right)$, let $y_{j}^{i}$ be the node mapped to $p^{i}$ at which $f_{i}$ and $f_{0}$ have multiplicity $m_{j}$. The gluing theorem shows that one can smooth each node $y_{j}^{i}$ in $m_{j}$ ways to produce $\left(\prod m_{j}\right)^{2}$ maps in $\mathscr{L}_{m, f, \lambda}$, so

$$
\left|\mathscr{E}_{m, f, \lambda}\right|=\left(\prod m_{j}\right)^{2} \quad(\lambda \neq 0) .
$$

In order to prove (3-4), we use the following fact on stable maps. An irreducible component of a stable holomorphic map $f$ is a ghost component if its image is a point. Write the domain of $f$ as $C^{g} \cup C$ where $C^{g}$ is a connected curve whose irreducible components are all ghost components. Then the stability of $f$ implies that

$$
\chi\left(C^{g}\right)-\ell^{g}-n \leq-1
$$

where $\ell^{g}=\left|C^{g} \cap C\right|$ and $n$ is the number of marked points on $C^{g}$.

Lemma 3.1. Let $\mathcal{M}_{r}$ and $\mathcal{M}_{m, 0}$ be as above. Then we have

$$
\lim _{\lambda \rightarrow 0} M_{\lambda} \subset \bigcup_{m} M_{m, 0}
$$

where the union is over all partitions $m$ of $d$ with $d-\ell(m)$ even.

Proof. Let $f$ be a limit map in (3-3). The domain $C$ of $f$ can be written as

$$
C=C_{1} \cup C_{0} \cup C_{2} \cup\left(\bigcup_{i=1}^{k+3} C_{i}^{g}\right) \cup C^{g} \cup \widetilde{C}^{g}
$$

where $C_{0}$ maps to $E, C_{1}$ and $C_{2}$ map to $D_{1}$ and $D_{2}, C_{i}^{g}$ is the union of all ghost components over $q^{i}$, where $i=1, \ldots, k+3, C^{g}$ is the union of all ghost components over points in $D_{0} \backslash\left(V_{1} \cup V_{0} \cup V_{2}\right)$, and $\widetilde{C}^{g}$ is the union of all ghost components over $\left\{p^{1}, p^{2}\right\}$. Let $f_{j}=\left.f\right|_{C_{j}}$ for $j=0,1,2$. Observe that $f_{j}$ is $V_{j}$-regular because $C_{j}$ has no ghost components. Let $\widehat{m}^{i}$ be a contact vector over $q^{i}, \widetilde{m}^{1}$ and $\widetilde{m}^{2}$ be contact vectors of $f_{1}$ and $f_{2}$ over $p^{1}$ and $p^{2}$, and $\widetilde{m}^{0 ; 1}$ and $\widetilde{m}^{0 ; 2}$ be contact vectors of $f_{0}$ over $p^{1}$ and $p^{2}$. The Riemann-Hurwitz formulas for $f_{0}, f_{1}$, and $f_{2}$ give

$$
\sum_{j=0}^{2} \chi\left(C_{j}\right) \leq 2 d(1-h)+\sum_{i=1}^{k+3}\left(\ell\left(\widehat{m}^{i}\right)-d\right)+\sum_{i=1}^{2}\left(\ell\left(\widetilde{m}^{i}\right)+\ell\left(\widetilde{m}^{0 ; i}\right)\right) .
$$


For $i=1, \ldots, k+3$, let $\ell_{i}=\left|C_{1} \cup C_{0} \cup C_{2} \cap C_{i}^{g}\right|$ and let $n_{i}$ be the number of marked points on $C_{i}^{g}$. Since all marked points are limits of marked points, we have

$$
\ell\left(\widehat{m}^{i}\right)=\ell\left(m^{i}\right)-n_{i}+\ell_{i} .
$$

For $j=0,1,2$, let $\tilde{\ell}_{j}=\left|C_{j} \cap \widetilde{C}^{g}\right|$. Counting the number of nodes mapped to $p^{1}$ and $p^{2}$ shows

$$
\sum_{i=1}^{2}\left(\ell\left(\tilde{m}^{i}\right)-\tilde{\ell}_{i}\right)=\sum_{i=1}^{2}\left|C_{i} \cap C_{0}\right|=\sum_{i=1}^{2} \ell\left(\tilde{m}^{0 ; i}\right)-\tilde{\ell}_{0} .
$$

Let $\ell^{g}=\left|C_{1} \cup C_{0} \cup C_{2} \cap C^{g}\right|$. Since $\chi(C)=\chi$, by (3-10) and (3-13) we have

$$
\begin{aligned}
\chi=\sum_{j=0}^{2} \chi\left(C_{j}\right)+\sum_{i=1}^{k+3}\left(\chi\left(C_{i}^{g}\right)-2 \ell_{i}\right) & +\chi\left(C^{g}\right)-2 \ell^{g} \\
& +\chi\left(\widetilde{C}^{g}\right)-\tilde{\ell}-\sum_{i=1}^{2}\left(\ell\left(\tilde{m}^{i}\right)+\ell\left(\tilde{m}^{0 ; i}\right)\right),
\end{aligned}
$$

where $\tilde{\ell}=\tilde{\ell}_{0}+\tilde{\ell}_{1}+\tilde{\ell}_{2}$. By our assumption that formula (0-1) holds, it follows from (3-11), (3-12), and (3-14) that

$$
\chi \leq \chi+\sum_{i=1}^{k+3}\left(\chi\left(C_{i}^{g}\right)-\ell_{i}-n_{i}\right)+\chi\left(C^{g}\right)-2 \ell^{g}+\chi\left(\widetilde{C}^{g}\right)-\tilde{\ell} .
$$

Noting that $C^{g}$ and $\widetilde{C}^{g}$ have no marked points, by (3-9) and (3-15), we conclude that the domain $C$ of $f$ has no ghost components. Consequently,

- $f_{j}$ is $V_{j}$-regular for $j=0,1,2$,

- $\widetilde{m}^{i}=\widetilde{m}^{0 ; i}$ for $i=1,2$ (see Lemma 3.3 of [Ionel and Parker 2004]) and $\widehat{m}^{i}=m^{i}$ for $i=1, \ldots, k+3$.

In particular, the equality in (3-11) holds; otherwise we have a strict inequality in (3-15). So, we have $\chi\left(C_{0}\right)=\ell\left(\tilde{m}^{1}\right)+\ell\left(\tilde{m}^{2}\right)$. But $\chi\left(C_{0}\right) \leq 2 \min \left\{\ell\left(\widetilde{m}^{1}\right), \ell\left(\tilde{m}^{2}\right)\right\}$. It follows that

- $C_{0}$ has $\ell\left(\tilde{m}^{1}\right)=\ell\left(\tilde{m}^{2}\right)$ connected components $E_{j}$ with $\chi\left(E_{j}\right)=2$ for all $j$,

- $\tilde{m}_{j}^{1}=\operatorname{deg}\left(\left.f_{0}\right|_{E_{j}}\right)=\tilde{m}_{j}^{2}$ for all $j$, that is, $\tilde{m}^{1}=\tilde{m}^{2}$.

It follows that the Euler characteristics of $C_{0}, C_{1}$, and $C_{2}$ satisfy (2-1) by (3-14). Therefore, $f \in \mathcal{M}_{m, 0}$ for $m=\widetilde{m}^{1}=\widetilde{m}^{2}$ and $d-\ell(m)$ is even by Lemma 2.1.

\section{Smooth model by Schiffer variation}

A Schiffer variation of a nodal curve (compare [Arbarello et al. 2011, p. 184]) is obtained by gluing deformations $u v=\lambda$ near nodes with the trivial deformation 
away from nodes. In this section, we use the method of Schiffer variation to construct a smooth model for the space $\mathscr{E}_{m, f}$ in (3-5), which has several branches intersecting at $f$ unless $m$ is trivial.

In this section, we fix an odd partition $m=\left(n^{\ell}\right)$, that is, $m=\left(m_{1}, \ldots, m_{\ell}\right)$ with

$$
m_{1}=\cdots=m_{\ell}=n, \quad \text { where } n=d / \ell \text { is odd. }
$$

Let $f=\left(f_{1}, f_{0}, f_{2}\right)$ be a map in $M_{m, 0}$ in (2-2). As described in Section 2, the central fiber of $\rho: \mathscr{D} \rightarrow \Delta$ is the nodal curve $D_{0}=D_{1} \cup E \cup D_{2}$ with two nodes $p^{1} \in D_{1} \cap E$ and $p^{2} \in D_{2} \cap E$ where $E=\mathbb{P}^{1}$. The domain of $f$ is a nodal curve

$$
C=C_{1} \cup C_{0} \cup C_{2}, \quad \text { where } C_{0}=\bigcup_{j=1}^{\ell} E_{\ell},
$$

with $2 \ell$ nodes, such that, for $i=1,2$ and $j=1, \ldots, \ell$,

- $f^{-1}\left(p^{i}\right)$ consists of the $\ell$ nodes $y_{j}^{i} \in C_{i} \cap E_{j}$,

- $C_{i}$ is smooth and $\left.f\right|_{C_{i}}=f_{i}$ has ramification index $m_{j}=n$ at the node $y_{j}^{i}$,

- $E_{j}=\mathbb{P}^{1}$ and $\left.f\right|_{E_{j}}=\left.f_{0}\right|_{E_{j}}: E_{j} \rightarrow E$ has ramification index $m_{j}=n$ at the node $y_{j}^{i}$.

The following is the main result of this section.

Proposition 4.1. Let $f$ be as above. Then, for each vector $\zeta=\left(\zeta_{1}^{1}, \zeta_{1}^{2}, \ldots, \zeta_{\ell}^{1}, \zeta_{\ell}^{2}\right)$, where $\zeta_{j}^{i}$ is an $n^{\text {th }}$ root of unity, there are a family of curves $\varphi_{\zeta}: \mathscr{C}_{\zeta} \rightarrow \Delta$, with smooth total space $\mathscr{C}_{\zeta}$, over a disk $\Delta$ (with parameter $s$ ) and a holomorphic map $\mathscr{F}_{\zeta}: \mathscr{C}_{\zeta} \rightarrow$ D satisfying:

(a) the central fiber $C_{\zeta, 0}=C$ and the restriction map $\left.\mathscr{F}_{\zeta}\right|_{C}=f$;

(b) the general fiber $C_{\zeta, s}(s \neq 0)$ is smooth and, for $\lambda=s^{n} \neq 0$,

$$
\bigcup_{\zeta}\left\{f_{\zeta, s}\right\}=\mathscr{E}_{m, f, \lambda}
$$

where the union is over all $\zeta, f_{\zeta, s}=\left.\mathscr{F}_{\zeta}\right|_{C_{\zeta, s}}$ and $\mathscr{E}_{m, f, \lambda}$ is the space (3-6).

Proof. The proof consists of four steps.

Step 1 . We first show how to construct the family of curves $\rho: \mathscr{D} \rightarrow \Delta$ with $k+3$ sections. For $i=1,2$, a neighborhood of the node $p^{i} \in D_{i} \cap E$ can be regarded as the union $U^{i} \cup V^{i}$ of the two disks

$$
U^{i}=\left\{u^{i} \in \mathbb{C}:\left|u^{i}\right|<1\right\} \subset D_{i} \quad \text { and } \quad V^{i}=\left\{v^{i} \in \mathbb{C}:\left|v^{i}\right|<1\right\} \subset E
$$

with their origins identified. We may assume that the fixed points $q^{1}, \ldots, q^{k+3}$ in $D_{0}$ described in (2-1) lie outside these sets. Consider the regions 
where

$$
\begin{aligned}
A^{i} & =\left\{\left(u^{i}, v^{i}, \lambda\right) \in U^{i} \times V^{i} \times \Delta: u^{i} v^{i}=\lambda\right\}, \\
B & =\bigcup_{i=1}^{2} G^{i} \cup\left[\left(D_{0} \backslash \bigcup_{i=1}^{2}\left(U^{i} \cup V^{i}\right)\right) \times \Delta\right],
\end{aligned}
$$

$$
G^{i}=\left\{\left(u^{i}, \lambda\right) \in U^{i} \times \Delta:\left|u^{i}\right|>\sqrt{|\lambda|}\right\} \cup\left\{\left(v^{i}, \lambda\right) \in V^{i} \times \Delta:\left|v^{i}\right|>\sqrt{|\lambda|}\right\} .
$$

We obtain a smooth complex surface $\mathscr{D}$ by gluing $A^{1}, A^{2}$, and $B_{0}$ using the maps

$$
G^{i} \rightarrow A^{i} \quad \text { defined by }\left(u^{i}, \lambda\right) \rightarrow\left(u^{i}, \frac{\lambda}{u^{i}}, \lambda\right) \text { and }\left(v^{i}, \lambda\right) \rightarrow\left(\frac{\lambda}{v^{i}}, v^{i}, \lambda\right) .
$$

Let $\rho: \mathscr{D} \rightarrow \Delta$ be the projection to the last factor and define $k+3$ sections $Q^{i}$ of $\rho$ by

$$
Q^{i}(\lambda)=\left(q^{i}, \lambda\right)
$$

Step 2. We can similarly construct a family of curves over a $2 \ell$-dimensional polydisk:

$$
\varphi_{2 \ell}: \mathscr{X} \rightarrow \Delta_{2 \ell}=\left\{t=\left(t_{1}^{1}, t_{1}^{2}, \ldots, t_{\ell}^{1}, t_{\ell}^{2}\right) \in \mathbb{C}^{2 \ell}:\left|t_{j}^{i}\right|<1\right\} .
$$

For each node $y_{j}^{i} \in C_{i} \cap E_{j}$, choose a neighborhood obtained from two disks

$$
U_{j}^{i}=\left\{u_{j}^{i} \in \mathbb{C}:\left|u_{j}^{i}\right|<1\right\} \subset C_{i} \quad \text { and } \quad V_{j}^{i}=\left\{v_{j}^{i} \in \mathbb{C}:\left|v_{j}^{i}\right|<1\right\} \subset E_{j}
$$

by identifying the origins. Consider the regions

$$
\begin{aligned}
A_{j}^{i} & =\left\{\left(u_{j}^{i}, u_{j}^{i}, t\right) \in U_{j}^{i} \times V_{j}^{i} \times \Delta_{2 \ell}: u_{j}^{i} v_{j}^{i}=t_{j}^{i}\right\}, \\
B_{2 \ell} & =\bigcup_{i, j} G_{j}^{i} \cup\left[\left(C \backslash \bigcup_{i, j}\left(U_{j}^{i} \cup V_{j}^{i}\right)\right) \times \Delta_{2 \ell}\right],
\end{aligned}
$$

where

$$
G_{j}^{i}=\left\{\left(u_{j}^{i}, t\right) \in U_{j}^{i} \times \Delta_{2 \ell}:\left|u_{j}^{i}\right|>\sqrt{\left|t_{j}^{i}\right|}\right\} \cup\left\{\left(v_{j}^{i}, t\right) \in V_{j}^{i} \times \Delta_{2 \ell}:\left|v_{j}^{i}\right|>\sqrt{\left|t_{j}^{i}\right|}\right\} .
$$

We can then obtain a smooth complex manifold $\mathscr{X}$ of dimension $2 \ell+1$ by gluing $\bigcup A_{j}^{i}$ and $B_{2 \ell}$ with the maps

$$
G_{j}^{i} \rightarrow A_{j}^{i} \quad \text { defined by }\left(u_{j}^{i}, t\right) \rightarrow\left(u_{j}^{i}, \frac{t_{j}^{i}}{u_{j}^{i}}, t\right) \text { and }\left(v_{j}^{i}, t\right) \rightarrow\left(\frac{t_{j}^{i}}{v_{j}^{i}}, v_{j}^{i}, t\right) \text {. }
$$

Let $\varphi_{2 \ell}: \mathscr{Q} \rightarrow \Delta$ be the projection to the factor $t$.

Step 3. Since $f_{i}$ and $\left.f_{0}\right|_{E_{j}}$ have ramification index $m_{j}=n$ at $y_{j}^{i}$, we may assume (after coordinates change) that on $U_{j}^{i}$ and $V_{j}^{i}$ the map $f$ can be written as

$$
U_{j}^{i} \rightarrow U^{i} \text { by } u_{j}^{i} \rightarrow\left(u_{j}^{i}\right)^{n} \quad \text { and } \quad V_{j}^{i} \rightarrow V^{i} \text { by } v_{j}^{i} \rightarrow\left(v_{j}^{i}\right)^{n} .
$$


For each $i, j$, define a map

$$
G_{j}^{i} \rightarrow G^{i} \quad \text { by }\left(u_{j}^{i}, t\right) \rightarrow\left(\left(u_{j}^{i}\right)^{n},\left(t_{j}^{i}\right)^{n}\right) \text { and }\left(u_{j}^{i}, t\right) \rightarrow\left(\left(v_{j}^{i}\right)^{n},\left(t_{j}^{i}\right)^{n}\right) .
$$

On the other hand, for each $i, j$, we have a map

$$
A_{j}^{i} \rightarrow A^{i} \quad \text { defined by }\left(u_{j}^{i}, v_{j}^{i}, t\right) \rightarrow\left(\left(u_{j}^{i}\right)^{n},\left(v_{j}^{i}\right)^{n},\left(t_{j}^{i}\right)^{n}\right) .
$$

These two maps (4-7) and (4-8) are glued together under the maps (4-3) and (4-5). The glued map extends to a holomorphic map $f_{t}: \mathscr{X}_{t} \rightarrow D_{\lambda}$ if and only if

$$
\left(t_{1}^{1}\right)^{n}=\left(t_{1}^{2}\right)^{n}=\cdots=\left(t_{\ell}^{1}\right)^{n}=\left(t_{\ell}^{2}\right)^{n}=\lambda .
$$

There are $n^{2 \ell}$ solutions $t$ of (4-9) and the extension map $f_{t}$ is given by

$$
(x, t) \rightarrow(f(x), \lambda) \quad \text { on } \mathscr{X}_{t}-\bigcup A_{j}^{i} .
$$

Step 4. For each vector $\zeta=\left(\zeta_{1}^{1}, \zeta_{1}^{2}, \ldots, \zeta_{\ell}^{1}, \zeta_{\ell}^{2}\right)$, where each $\zeta_{j}^{i}$ is an $n^{\text {th }}$ root of unity, define

$$
\delta_{\zeta}: \Delta \rightarrow \Delta_{2 \ell} \text { by } s \rightarrow\left(\zeta_{1}^{1} s, \zeta_{1}^{2} s, \zeta_{2}^{1} s, \zeta_{2}^{2} s, \ldots, \zeta_{\ell}^{1} s, \zeta_{\ell}^{2} s\right)
$$

The pullback $\delta_{\zeta}^{*} \mathscr{X}$ gives a family of curves:

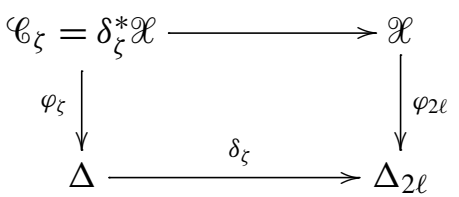

The central fiber is $C_{\zeta, 0}=C$ and the general fiber $C_{\zeta, s}(s \neq 0)$ is smooth. A neighborhood of the node $y_{j}^{i}$ of $C$ in $\mathscr{C}_{\zeta}$ can be viewed as

$$
\hat{A}_{j}^{i}=\left\{\left(u_{j}^{i}, v_{j}^{i}, s\right) \in \mathbb{C}^{3}:\left|u_{j}^{i}\right|<1,\left|v_{j}^{i}\right|<1, u_{j}^{i} v_{j}^{i}=\zeta_{j}^{i} s\right\} .
$$

It follows that the total space $\mathscr{b}_{\zeta}$ is a complex smooth surface. Noting $\delta_{\zeta}(s)$ is a solution of (4-9) for $\lambda=s^{n}$, we obtain a holomorphic map $\mathscr{F}_{\zeta}: \mathscr{C}_{\zeta} \rightarrow \mathscr{D}$ given by

$$
\begin{aligned}
\left(u_{j}^{i}, v_{j}^{i}, s\right) & \rightarrow\left(\left(u_{j}^{i}\right)^{n},\left(v_{j}^{i}\right)^{n}, s^{n}\right) & & \text { on } \hat{A}_{j}^{i}, \\
(x, s) & \rightarrow\left(f(x), s^{n}\right) & & \text { on } \mathscr{C}_{\zeta}-\bigcup \hat{A}_{j}^{i} .
\end{aligned}
$$

Since the restriction $\left.\mathscr{F}_{\zeta}\right|_{C}=f$ by (4-6) and (4-12), it remains to show (4-2). By our choice of fixed points $q^{i}$ on $D_{0}$, each contact marked point $x_{j}^{i}$ of $f$ lies in $\mathscr{C}_{\zeta}-\bigcup \hat{A}_{j}^{i}$. Thus, by (4-12), the pullback $\mathscr{F}_{\zeta}^{*} Q^{i}$ of the section $Q^{i}$ of $\rho$ gives a section $X_{j}^{i}$ of $\varphi_{\zeta}$ given by $X_{j}^{i}(s)=\left(x_{j}^{i}, s\right)$. After marking the points $X_{j}^{i}(s)$ in $C_{\zeta, s}$, the restriction map

$$
f_{\zeta, s}=\left.\mathscr{F}_{\zeta}\right|_{C_{\zeta, s}}: C_{\zeta, s} \rightarrow D_{\lambda}, \quad \text { where } \lambda=s^{n} \neq 0,
$$


has contact marked points $X_{j}^{i}(s)$ over $Q^{i}(\lambda)$ with multiplicity $m_{j}^{i}$. This means that $f_{\zeta, s}$ lies in the space $M_{\lambda}$ in (3-2) for $\lambda=s^{n}$. Therefore, noting that $f_{\zeta, s} \rightarrow f$ as $s \rightarrow 0$ and that $\left|\mathscr{E}_{m, f, \lambda}\right|=n^{2 \ell}$ by (3-8), we conclude (4-2).

\section{Spin structure and parity}

The aim of this section is to use a spin structure on a family of nodal curves [Cornalba 1989] to show the parity calculation in Proposition 5.4. Twisting a bundle as in (5-6) is a key idea for parity calculation.

We first introduce a spin structure on a family of nodal curves that is relevant to our discussion. We refer to [Cornalba 1989] for the definition of spin structure and more details. The relative dualizing sheaf $\omega_{\rho}$ of the family of curves $\rho: \mathscr{D} \rightarrow \Delta$ in (3-1) is the canonical bundle $K_{\mathscr{D}}$ on the total space $\mathscr{D}$, since $\mathscr{D}$ is smooth and $K_{\Delta}$ is trivial. For each $\lambda \neq 0$, the restriction $\left.K_{\mathscr{D}}\right|_{D_{\lambda}}$ is the canonical bundle $K_{D_{\lambda}}$ on $D_{\lambda}$, and the restriction $\left.K_{\mathscr{D}}\right|_{D_{0}}$ is the dualizing sheaf $\omega_{D_{0}}$ of the nodal curve $D_{0}=D_{1} \cup E \cup D_{2}$. As described in Section $4, D_{0}$ is locally given by $u^{i} v^{i}=0$ near each node $p^{i}$ in $D_{i} \cap E$ for $i=1,2$. Then the local generators of $\omega_{D_{0}}$ are $d u^{i} / u^{i}$ and $d v^{i} / v^{i}$ with a relation $d u^{i} / u^{i}+d v^{i} / v^{i}=0$; see [Harris and Morrison 1998, p. 82]. This implies the restriction $\left.\omega_{D_{0}}\right|_{D_{i}}=K_{D_{i}} \otimes \mathcal{O}\left(p^{i}\right)$. On the other hand, $1 / u^{i}$ is a local defining function for the divisor $-E$ on $\mathscr{D}$ near $p^{i}$. By restricting $1 / u^{i}$ to $D_{i}$, one can see that $\left.\mathcal{O}(-E)\right|_{D_{i}}=\mathcal{O}\left(-p^{i}\right)$. Consequently, for $i=1,2$,

$$
\left.\left.K_{\mathscr{D}}\right|_{D_{i}} \otimes \mathcal{O}(-E)\right|_{D_{i}}=\left.\omega_{D_{0}}\right|_{D_{i}} \otimes \mathcal{O}\left(-p^{i}\right)=K_{D_{i}} .
$$

From Cornalba's construction [1989, p. 570], there are a line bundle $\mathcal{N} \rightarrow \mathscr{D}$ and a homomorphism $\Phi: \mathcal{N}^{2} \rightarrow \omega_{\rho}=K_{\mathscr{D}}$ satisfying the following.

- $\Phi$ vanishes identically on the exceptional component $E$ and $\left.\mathcal{N}\right|_{E}=\mathscr{O}_{E}(1)$.

- Since $\left.\Phi\right|_{E} \equiv 0$, there is an induced homomorphism $\hat{\Phi}: \mathcal{N}^{2} \rightarrow K_{\mathscr{D}} \otimes \mathcal{O}(-E)$ such that $\Phi$ is the composition of $\hat{\Phi}$ with tensoring with $\eta$ :

$$
\Phi: \mathcal{N}^{2} \stackrel{\hat{\Phi}}{\longrightarrow} K_{\mathscr{D}} \otimes \mathcal{O}(-E) \stackrel{\otimes \eta}{\longrightarrow} K_{\mathscr{D}},
$$

where $\eta$ is a section of $O(E)$ with zero divisor $E$. Then, for $i=1,2$, the restriction

$$
\left.\hat{\Phi}\right|_{D_{i}}:\left.\left.\left(\left.\mathcal{N}\right|_{D_{i}}\right)^{2} \rightarrow K_{\mathscr{D}}\right|_{D_{i}} \otimes \mathcal{O}(-E)\right|_{D_{i}}=K_{D_{i}}
$$

is an isomorphism so that the restriction $N_{i}=\left.\mathcal{N}\right|_{D_{i}}$ is a theta characteristic on $D_{i}$.

- For each $\lambda \neq 0$, the restriction $\left.\Phi\right|_{D_{\lambda}}:\left(\left.\mathcal{N}\right|_{D_{\lambda}}\right)^{2} \rightarrow K_{D_{\lambda}}$ is an isomorphism so that the restriction $N_{\lambda}=\left.\mathcal{N}\right|_{D_{\lambda}}$ is a theta characteristic on $D_{\lambda}$.

The pair $(\mathcal{N}, \Phi)$ is a spin structure on $\rho: \mathscr{D} \rightarrow \Delta$ and the restriction $\left.\mathcal{N}\right|_{D_{0}}$ is a theta characteristic on the nodal curve $D_{0}$. 
Remark 5.1. Atiyah [1971] and Mumford [1971] showed that the parity of a theta characteristic on a smooth curve is a deformation invariant. Cornalba [1989, Page 580] used the homomorphism $\Phi$ to extend Mumford's proof to the case of spin structure on a family of nodal curves. Thus, if $p_{1}, p_{2}$, and $p$ are the parities of $N_{1}, N_{2}$, and $N_{\lambda}(\lambda \neq 0)$, we have

$$
p \equiv p_{1}+p_{2}(\bmod 2) .
$$

Let $\varphi_{\zeta}: \mathscr{C}_{\zeta} \rightarrow \Delta$ be the family of curves in Proposition 4.1. Recall that the central fiber of $\varphi_{\zeta}$ is $C=C_{1} \cup C_{0} \cup C_{2}$, where $C_{0}=\bigsqcup_{j} E_{j}$ is a disjoint union of $\ell$ exceptional components $E_{j}$ and $C_{i} \cap E_{j}=\left\{y_{j}^{i}\right\}$ for $i=1,2$ and $1 \leq j \leq \ell$. Similarly as for (5-1), by restricting local defining functions, we have

$$
\left.\mathcal{O}\left( \pm C_{0}\right)\right|_{C_{i}}=\mathcal{O}\left( \pm \sum_{j} y_{j}^{i}\right) \quad(i=1,2) \quad \text { and }\left.\quad \mathcal{O}\left( \pm C_{0}\right)\right|_{C_{\zeta, s}}=\mathcal{O} \quad(s \neq 0) .
$$

Since any fiber of $\varphi_{\zeta}$ is a principal divisor on $\mathscr{C}_{\zeta}, \mathcal{O}(C)=\mathbb{O}$ and hence $\mathscr{O}\left(C_{0}\right)=$ $\mathrm{O}\left(-C_{1}-C_{2}\right)$. We also have

$$
\left.\mathcal{O}\left( \pm C_{0}\right)\right|_{E_{j}}=\left.\mathcal{O}\left(\mp\left(C_{1}+C_{2}\right)\right)\right|_{E_{j}}=\mathcal{O}\left(\mp\left(y_{j}^{1}+y_{j}^{2}\right)\right)=\mathscr{O}(\mp 2)(1 \leq j \leq \ell) .
$$

Let $f=\left(f_{1}, f_{0}, f_{2}\right)$ and $\mathscr{F}_{\zeta}: \mathscr{C}_{\zeta} \rightarrow \mathscr{D}$ be the maps in Proposition 4.1. The ramification divisor $R_{\mathscr{F}_{\zeta}}$ of $\mathscr{F}_{\zeta}$ has local defining functions given by the Jacobian of $\mathscr{F}_{\zeta}$, so (4-12) shows

$$
R_{\mathscr{F}_{\zeta}}=\mathcal{O}\left(X_{\zeta}+(n-1) C\right)=\mathcal{O}\left(X_{\zeta}\right),
$$

where $X_{\zeta}=\sum_{i, j}\left(m_{j}^{i}-1\right) X_{j}^{i}$ and $X_{j}^{i}$ is the section of $\varphi_{\zeta}$ defined in (4-12). Note that

(i) the ramification divisor of $f_{i}=\left.\mathscr{F}_{\zeta}\right|_{C_{i}}(i=1,2)$ is $R_{f_{i}}=\left.X_{\zeta}\right|_{C_{i}}+\sum_{j}(n-1) y_{j}^{i}$;

(ii) the ramification divisor of $f_{\zeta, s}=\left.\mathscr{F}_{\zeta}\right|_{C_{\zeta, s}}(s \neq 0)$ is $R_{f_{\zeta, s}}=\left.X_{\zeta}\right|_{C_{\zeta, s}}$.

Now, noting $n$ is odd, we twist the pullback bundle $\mathscr{F}_{\zeta}^{*} \mathcal{N}$ by setting

$$
\mathscr{L}_{\zeta}=\mathscr{F}_{\zeta}^{*} \mathcal{N} \otimes \mathcal{O}\left(\frac{1}{2} X_{\zeta}+\frac{(n-1)}{2} C_{0}\right)
$$

The lemma below shows that the twisted line $\mathscr{L}_{\zeta}$ restricts to a theta characteristic on each fiber of $\varphi_{\zeta}$, including the central fiber $C$.

Lemma 5.2. Let $\mathscr{L}_{\zeta}$ be as above. Then:

(a) $\left.\mathscr{L}_{\zeta}\right|_{E_{j}}=0$ (1) for $1 \leq j \leq \ell$.

(b) $\left.\mathscr{L}_{\zeta}\right|_{C_{1}}=L_{f_{1}},\left.\mathscr{L}_{\zeta}\right|_{C_{2}}=L_{f_{2}}$ and $\left.\mathscr{L}_{\zeta}\right|_{C_{\zeta, s}}=L_{f_{\zeta, s}}$ for $s \neq 0$, where $L_{f_{1}}, L_{f_{2}}, L_{f_{\zeta, s}}$ are the theta characteristics on $C_{1}, C_{2}, C_{\zeta, s}$ defined by (0-2). 
Proof. Part (a) follows from (5-4) and the fact that each restriction map $\left.\mathscr{F}_{\zeta}\right|_{E_{j}}$ has degree $n$. Part (b) follows from (5-3), (i), and (ii).

Observe that the relative dualizing sheaf $\omega_{\varphi_{\zeta}}$ is the canonical bundle $K_{\mathscr{C}_{\zeta}}$ since $\mathscr{C}_{\zeta}$ is smooth. The Hurwitz formula and (5-5) thus imply that

$$
\omega_{\varphi_{\zeta}}=K_{\mathscr{C}_{\zeta}}=\mathscr{F}_{\zeta}^{*} K_{\mathscr{D}} \otimes \mathcal{O}\left(X_{\zeta}\right) .
$$

Define a homomorphism

$$
\begin{aligned}
\hat{\Psi}_{\zeta}: \mathscr{L}_{\zeta}^{2}=\mathscr{F}_{\zeta}^{*} \mathcal{N}^{2} \otimes \mathcal{O}\left(X_{\zeta}+(n-1) C_{0}\right) & \\
& \rightarrow \mathscr{F}_{\zeta}^{*}\left(K_{\mathscr{D}} \otimes \mathcal{O}(-E)\right) \otimes \mathcal{O}\left(X_{\zeta}+(n-1) C_{0}\right)
\end{aligned}
$$

by $\hat{\Psi}_{\zeta}=\mathscr{F}_{\zeta}^{*} \hat{\Phi} \otimes \mathrm{Id}$, where $\hat{\Phi}$ is the induced homomorphism in (5-2). Noting that $\mathcal{O}(C)=\mathcal{O}$ and $\mathcal{O}\left(D_{0}\right)=\mathcal{O}$, by $(4-12)$, we have

$$
\mathscr{F}_{\zeta}^{*} \mathcal{O}(-E)=\mathscr{F}_{\zeta}^{*} \mathcal{O}\left(D_{1}+D_{2}\right)=\mathcal{O}\left(n\left(C_{1}+C_{2}\right)\right)=\mathscr{O}\left(-n C_{0}\right) .
$$

Together with (5-7), this implies that the right side of (5-8) is $K_{\mathscr{C}_{\zeta}} \otimes \mathcal{O}\left(-C_{0}\right)$. Now define a homomorphism $\Psi_{\zeta}: \mathscr{L}_{\zeta}^{2} \rightarrow K_{\mathscr{C}_{\zeta}}$ to be the composition

$$
\Psi_{\zeta}: \mathscr{L}_{\zeta}^{2} \stackrel{\hat{\Psi}_{\zeta}}{\longrightarrow} K_{\mathscr{C}_{\zeta}} \otimes \mathcal{O}\left(-C_{0}\right) \stackrel{\otimes \xi}{\longrightarrow} K_{\mathscr{C}_{\zeta}},
$$

where $\xi$ is a section of $\mathcal{O}\left(C_{0}\right)$ with zero divisor $C_{0}$.

Lemma 5.3. $\left(\mathscr{L}_{\zeta}, \Psi_{\zeta}\right)$ is a spin structure on $\varphi_{\zeta}: \mathscr{C}_{\zeta} \rightarrow \Delta$.

Proof. First, $\left.\mathscr{L}_{\zeta}\right|_{E}=\mathcal{O}(1)$ by Lemma 5.2(a) and $\Psi_{\zeta}$ vanishes identically on each exceptional component $E_{j}$, since $\xi=0$ on $C_{0}=\bigsqcup_{j} E_{j}$. Second, since $\left.\hat{\Phi}\right|_{D_{i}}$ is an isomorphism, (5-3) and (i) show that, for $i=1,2$, the restriction

$$
\left.\hat{\Psi}\right|_{C_{i}}=f_{i}^{*}\left(\left.\hat{\Phi}\right|_{D_{i}}\right) \otimes \operatorname{Id}:\left(\left.\mathscr{L}_{\zeta}\right|_{C_{i}}\right)^{2}=f_{i}^{*} N_{i}^{2} \otimes \mathcal{O}\left(R_{f_{i}}\right) \rightarrow f_{i}^{*} K_{D_{i}} \otimes \mathcal{O}\left(R_{f_{i}}\right)=K_{C_{i}}
$$

is an isomorphism. Lastly, let $\lambda=s^{n} \neq 0$. Since $\left.\Phi\right|_{D_{\lambda}}$ is an isomorphism, so is $\left.\hat{\Phi}\right|_{D_{\lambda}}$. Thus, by (5-3), (ii), and the facts $\left.K_{\mathscr{D}}\right|_{D_{\lambda}}=K_{D_{\lambda}}$ and $\left.\mathcal{O}(-E)\right|_{D_{\lambda}}=\mathcal{O}$, the restriction

$\left.\hat{\Psi}_{\zeta}\right|_{C_{\zeta, s}}=\left.f_{\zeta, s}^{*} \hat{\Phi}\right|_{D_{\lambda}} \otimes \operatorname{Id}:\left(\left.\mathscr{L}_{\zeta}\right|_{C_{\zeta, s}}\right)^{2}=f_{\zeta, s}^{*} N_{\lambda}^{2} \otimes \mathcal{O}\left(R_{f_{\zeta, s}}\right) \rightarrow f_{\zeta, s}^{*} K_{D_{\lambda}} \otimes \mathcal{O}\left(R_{f_{\zeta, s}}\right)=K_{C_{\zeta, s}}$ is an isomorphism. This implies that the restriction

$$
\left.\Psi_{\zeta}\right|_{C_{\zeta, s}}:\left.\left(\left.\mathscr{L}_{\zeta}\right|_{C_{\zeta, s}}\right)^{2} \rightarrow K_{C_{\zeta}}\right|_{C_{\zeta, s}}=K_{C_{\zeta}, s}
$$

is also an isomorphism. Therefore, we conclude that $\left(\mathscr{L}_{\zeta}, \Psi_{\zeta}\right)$ is a spin structure on $\varphi_{\zeta}$.

The following is a key fact for the proof of Theorem 0.1 . 
Proposition 5.4. Let $f=\left(f_{1}, f_{0}, f_{2}\right)$ and $f_{\zeta, s}$ be maps in Proposition 4.1. Then, for all $s \neq 0$,

$$
p\left(f_{\zeta, s}\right) \equiv p\left(f_{1}\right)+p\left(f_{2}\right)(\bmod 2) .
$$

Proof. Since $\left(\mathscr{L}_{\zeta}, \Psi_{\zeta}\right)$ is a spin structure on $\varphi_{\zeta}$, Cornalba's proof, mentioned in Remark 5.1, shows that, for all $s \neq 0$,

$$
h^{0}\left(\left.\mathscr{L}_{\zeta}\right|_{C_{\zeta, s}}\right) \equiv h^{0}\left(\left.\mathscr{L}_{\zeta}\right|_{C_{1}}\right)+h^{0}\left(\left.\mathscr{L}_{\zeta}\right|_{C_{2}}\right)(\bmod 2) .
$$

This and Lemma 5.2(b) prove (5-10).

\section{Proof of Theorem 0.1}

Proof. Fix a spin structure $(\mathcal{N}, \Phi)$ on $\rho: \mathscr{D} \rightarrow \Delta$ given in Section 5. Consider the space $\mathcal{M}_{m, 0}$ in (2-2) where $m$ is a partition of $d=3$. In this case, by Lemma 2.1, either $m=\left(1^{3}\right)$ or $m=(3)$. Note that both of them satisfy (4-1). Fix $\lambda \neq 0$ and let $f=\left(f_{1}, f_{0}, f_{2}\right)$ be a map in $\mathcal{M}_{m, 0}$. Then (4-2) and (5-10) show that, for all $f_{\mu} \in \mathscr{E}_{m, f, \lambda}$,

$$
p\left(f_{\mu}\right) \equiv p\left(f_{1}\right)+p\left(f_{2}\right)(\bmod 2) .
$$

Lemma 1.1 and (3-7) show that

$$
\begin{aligned}
H_{(3)^{k}}^{h, p} & =H_{(3)^{k},\left(1^{3}\right)^{3}}^{h, p} \\
& =\frac{1}{(3 !)^{3}}\left(\sum_{f \in \mathcal{M}_{\left(1^{3}\right), 0}} \sum_{f_{\mu} \in \mathscr{L}_{\left(1^{3}\right), f, \lambda}}(-1)^{p\left(f_{\mu}\right)}+\sum_{f \in \mathcal{M}_{(3), 0}} \sum_{f_{\mu} \in \mathscr{L}_{(3), f, \lambda}}(-1)^{p\left(f_{\mu}\right)}\right) .
\end{aligned}
$$

By (3-8) and (6-1), (6-2) becomes

(6-3) $H_{(3)^{k}}^{h, p}=\sum_{f=\left(f_{1}, f_{0}, f_{2}\right) \in \mathcal{M}_{\left(1^{3}\right), 0}} \frac{(-1)^{p\left(f_{1}\right)+p\left(f_{2}\right)}}{(3 !)^{3}}+\sum_{f=\left(f_{1}, f_{0}, f_{2}\right) \in \mathcal{M}_{(3), 0}} \frac{3^{2}(-1)^{p\left(f_{1}\right)+p\left(f_{2}\right)}}{(3 !)^{3}}$. It then follows from Lemma 2.3 and (6-3) that

$$
\begin{aligned}
H_{(3)^{k}}^{h, p} & =\sum_{\left(f_{1}, f_{0}, f_{2}\right) \in \mathscr{P}_{\left(1^{3}\right)}} \frac{(-1)^{p\left(f_{1}\right)+p\left(f_{2}\right)}}{(3 !)^{5}}+\sum_{\left(f_{1}, f_{0}, f_{2}\right) \in \mathscr{P}_{(3)}} \frac{3^{2}(-1)^{p\left(f_{1}\right)+p\left(f_{2}\right)}}{(3 !)^{3}} \\
& =\frac{1}{(3 !)^{3}} \sum_{f_{1} \in \mathcal{M}_{\left(1^{3}\right)}^{1}}(-1)^{p\left(f_{1}\right)} \sum_{f_{2} \in \mathcal{M}_{\left(1^{3}\right)}^{2}}(-1)^{p\left(f_{2}\right)}+\frac{3}{(3 !)^{2}} \sum_{f_{1} \in \mathcal{M}_{(3)}^{1}}(-1)^{p\left(f_{1}\right)} \sum_{f_{2} \in \mathcal{M}_{(3)}^{2}}(-1)^{p\left(f_{2}\right)} \\
& =3 ! H_{(3)^{k_{1}}}^{h_{1}, p_{1}} \cdot H_{(3)^{k_{2}}}^{h_{2}, p_{2}}+3 H_{(3)^{k_{1}+1}}^{h_{1}, p_{1}} \cdot H_{(3)^{k_{2}+1}}^{h_{2}, p_{2}}
\end{aligned}
$$

the second equality follows from Lemma 2.2 and the last from Lemma 1.1. 


\section{Calculation}

Proposition 7.1. $H_{(3)^{k}}^{h, \pm}=3^{2 h-2}\left[(-1)^{k} 2^{k+h-1} \pm 1\right]$.

Proof. The proof consists of four steps.

Step 1 . We first show the following facts which we use in the computation below.

Lemma 7.2. (a) $H_{(3)^{0}}^{0,+}=H_{3}^{0,+}=\frac{1}{3 !}, \quad$ (b) $H_{(3)^{3}}^{0,+}=-\frac{1}{3}, \quad$ (c) $H_{(3)^{0}}^{1,+}=H_{3}^{1,+}=2$.

Proof. Consider the dimension-zero space $M_{\chi}^{V}\left(\mathbb{P}^{1}, 3\right)$ where $V=\varnothing$. The Euler characteristic $\chi=6$ by (0-1), and hence the space contains only one map $f: C \rightarrow \mathbb{P}^{1}$ where $C$ is a disjoint union of three rational curves and $|\operatorname{Aut}(f)|=3$ !. This shows (a). Let $(f, C)$ be a map in the dimension-zero space $M_{\chi,(3),(3),(3)}^{V}\left(\mathbb{P}^{1}, 3\right)$. Then $C$ is a connected curve of genus one and the theta characteristic $L_{f}$ on $C$ defined by $(0-2)$ is

$$
L_{f}=\mathcal{O}\left(-2 x_{1}+x_{2}+x_{3}\right)=\mathscr{O}\left(x_{1}-2 x_{2}+x_{3}\right)=\mathscr{O}\left(x_{1}+x_{2}-2 x_{3}\right),
$$

where $x_{1}, x_{2}$, and $x_{3}$ are ramification points of $f$. This implies $L_{f}^{3}=0$, and hence $L_{f}=0$ because $L_{f}^{2}=L_{f}^{3}=0$. We have $p(f)=1$. Therefore,

$$
H_{(3)^{3}}^{0,+}=-H_{(3)^{3}}^{0}=-\frac{1}{3},
$$

where $H_{(3)^{3}}^{0}$ denotes the (ordinary) Hurwitz number, which is calculated by using the character formula; see [Okounkov and Pandharipande 2006, (0.10)]. By Proposition 9.2 of [Lee and Parker 2007], the spin Hurwitz numbers $H_{d}^{h, p}$ are the dimension-zero local invariants of spin curve that count maps from possibly disconnected domains. Let $G W_{d}^{h, p}$ denote the dimension-zero local invariants of spin curve that count maps from connected domains. Then $H_{d}^{h, p}$ and $G W_{d}^{h, p}$ are related as follows:

$$
1+\sum_{d>0} H_{d}^{h, p} t^{d}=\exp \left(\sum_{d>0} G W_{d}^{h, p} t^{d}\right) .
$$

Now (c) follows from $G W_{1}^{1,+}=1, G W_{2}^{1,+}=1 / 2$, and $G W_{3}^{1,+}=4 / 3$; see Section 10 of [Lee and Parker 2007].

Step 2. In this step, we compute $H_{(3)^{k}}^{1,-}$. For a spin curve of genus one with trivial theta characteristic. It follows from formula (3.12) of [Eskin et al. 2008] that

$$
H_{(3)^{k}}^{1,-}=2^{-k}\left[\left(\boldsymbol{f}_{(3)}(21)\right)^{k}-\left(\boldsymbol{f}_{(3)}(3)\right)^{k}\right] .
$$

Here the central character $f_{(3)}$ can be written as

$$
\boldsymbol{f}_{(3)}=\frac{1}{3} \boldsymbol{p}_{3}+a_{2} \boldsymbol{p}_{1}^{2}+a_{1} \boldsymbol{p}_{1}+a_{0}
$$

for some $a_{i} \in \mathbb{Q}(0 \leq i \leq 2)$, and the supersymmetric functions $\boldsymbol{p}_{1}$ and $\boldsymbol{p}_{3}$ are defined 
by

$$
\boldsymbol{p}_{1}(m)=d-\frac{1}{24} \quad \text { and } \quad \boldsymbol{p}_{3}(m)=\sum_{j} m_{j}^{3}-\frac{1}{240},
$$

where $m=\left(m_{1}, \ldots, m_{\ell}\right)$ is a partition of $d$. For $k=0,1,(7-1)$ shows

$$
H_{(3)^{0}}^{1,-}=0 \quad \text { and } \quad H_{(3)}^{1,-}=-3 .
$$

Lemma 7.2(b), (7-2), and formula (0-6) give $H_{(3)^{2}}^{1,-}=3 H_{(3)}^{1,-} \cdot H_{(3)^{3}}^{0,+}=3$. Together with (7-1) and (7-2), this yields $f_{(3)}(21)=-4$ and $f_{(3)}(3)=2$. From this and (7-1) we have, for $k \geq 0$,

$$
H_{(3)^{k}}^{1,-}=(-1)^{k} 2^{k}-1 .
$$

Step 3. In this step, we compute $H_{(3)^{k}}^{h,+}$ for $h=0,1$. For $k \geq 1$, (7-2) and formula (0-6) give $H_{(3)^{k-1}}^{1,-}=3 H_{(3)}^{1,-} \cdot H_{(3)^{k}}^{0,+}=-3^{2} H_{(3)^{k}}^{0,+}$. Combining this with Lemma 7.2(a) we obtain, for $k \geq 0$,

$$
H_{(3)^{k}}^{0,+}=-\frac{1}{3^{2}}\left((-1)^{k-1} 2^{k-1}-1\right) .
$$

Lemma 7.2(c), (7-3), (7-4), and formula (0-6) show

$$
\begin{aligned}
& H_{(3)^{0}}^{2,+}=3 ! H_{(3)^{0}}^{1,-} \cdot H_{(3)^{0}}^{1,-}+3 H_{(3)}^{1,-} \cdot H_{(3)}^{1,-}=27, \\
& H_{(3)}^{2,+}=3 ! H_{(3)^{0}}^{1,-} \cdot H_{(3)}^{1,-}+3 H_{(3)}^{1,-} \cdot H_{(3)^{2}}^{1,-}=-27, \\
& H_{(3)^{0}}^{2,+}=3 ! H_{(3)^{0}}^{1,+} \cdot H_{(3)^{0}}^{1,+}+3 H_{(3)}^{1,+} \cdot H_{(3)}^{1,+}=24+3 H_{(3)}^{1,+} \cdot H_{(3)}^{1,+}, \\
& H_{(3)}^{2,+}=3 ! H_{(3)^{0}}^{1,+} \cdot H_{(3)}^{1,+}+3 H_{(3)}^{1,+} \cdot H_{(3)^{2}}^{1,+}=12 H_{(3)}^{1,+}+3 H_{(3)}^{1,+} \cdot H_{(3)^{2}}^{1,+}, \\
& H_{(3)^{2}}^{1,+}=3 ! H_{(3)^{0}}^{1,+} \cdot H_{(3)^{2}}^{0,+}+3 H_{(3)}^{1,+} \cdot H_{(3)^{3}}^{0,+}=4-H_{(3)}^{1,+} .
\end{aligned}
$$

It follows that $H_{(3)}^{1,+}=-1$. Hence, Lemma 7.2(c), (7-4), and formula (0-6) give

$$
H_{(3)^{k}}^{1,+}=3 ! H_{(3)^{0}}^{1,+} \cdot H_{(3)^{k}}^{0,+}+3 H_{(3)}^{1,+} \cdot H_{(3)^{k+1}}^{0,+}=(-1)^{k} 2^{k}+1 .
$$

Step 4. It remains to compute $H_{(3)^{k}}^{h, p}$ for $h \geq 2$. The formula (0-6) gives

$$
H_{(3)^{k}}^{h, p}=3 ! H_{(3)^{0}}^{h-1, p} \cdot H_{(3)^{k}}^{1,+}+3 H_{(3)}^{h-1, p} \cdot H_{(3)^{k+1}}^{1,+} .
$$

From this, we can deduce that, for $h \geq 2$,

$$
\begin{aligned}
& \left(\begin{array}{l}
H_{(3)^{k}}^{h, p} \\
H_{(3)^{h+1}}^{h, p}
\end{array}\right)=\left(\begin{array}{ll}
3 ! H_{(3)^{k}}^{1,+} & 3 H_{(3)^{k+1}}^{1,+} \\
3 ! H_{(3)^{k+1}}^{1,+} & 3 H_{(3)^{k+2}}^{1,+}
\end{array}\right)\left(\begin{array}{l}
H_{(3)^{0}}^{h-1, p} \\
H_{(3)}^{h-1, p}
\end{array}\right) \\
& =\left(\begin{array}{ll}
3 ! H_{(3)^{k}}^{1,+} & 3 H_{(3)^{k+1}}^{1,+} \\
3 ! H_{(3)^{k+1}}^{1,+} & 3 H_{(3)^{k+2}}^{1,+}
\end{array}\right)\left(\begin{array}{ll}
3 ! H_{(3)^{0}}^{1,+} & 3 H_{(3)}^{1,+} \\
3 ! H_{(3)}^{1,+} & 3 H_{(3)^{2}}^{1,+}
\end{array}\right)^{h-2}\left(\begin{array}{c}
H_{(3)^{0}}^{1, p} \\
H_{(3)}^{1, p}
\end{array}\right) .
\end{aligned}
$$


Therefore, (7-3), (7-5), and (7-6) complete the proof.

\section{References}

[Arbarello et al. 2011] E. Arbarello, M. Cornalba, and P. A. Griffiths, Geometry of algebraic curves, vol. II, Grundlehren der Mathematischen Wissenschaften 268, Springer, Heidelberg, 2011. MR 2012e:14059 Zbl 1235.14002

[Atiyah 1971] M. F. Atiyah, "Riemann surfaces and spin structures", Ann. Sci. École Norm. Sup. (4) 4 (1971), 47-62. MR 44 \#3350 Zbl 0212.56402

[Cornalba 1989] M. Cornalba, "Moduli of curves and theta-characteristics", pp. 560-589 in Lectures on Riemann surfaces (Trieste, 1987), edited by M. Cornalba et al., World Scientific, Teaneck, NJ, 1989. MR 91m:14037 Zbl 0800.14011

[Eskin et al. 2008] A. Eskin, A. Okounkov, and R. Pandharipande, "The theta characteristic of a branched covering", Adv. Math. 217:3 (2008), 873-888. MR 2008k:14065 Zbl 1157.14014

[Gunningham 2012] S. Gunningham, "Spin Hurwitz numbers and topological quantum field theory", preprint, 2012. arXiv 1201.1273

[Harris and Morrison 1998] J. Harris and I. Morrison, Moduli of curves, Graduate Texts in Mathematics 187, Springer, New York, 1998. MR 99g:14031 Zbl 0913.14005

[Ionel and Parker 2003] E.-N. Ionel and T. H. Parker, "Relative Gromov-Witten invariants", Ann. of Math. (2) 157:1 (2003), 45-96. MR 2004a:53112 Zbl 1039.53101

[Ionel and Parker 2004] E.-N. Ionel and T. H. Parker, "The symplectic sum formula for GromovWitten invariants”, Ann. of Math. (2) 159:3 (2004), 935-1025. MR 2006b:53110 Zbl 1075.53092

[Kiem and Li 2007] Y.-H. Kiem and J. Li, "Gromov-Witten invariants of varieties with holomorphic 2-forms”, preprint, 2007. arXiv 0707.2986

[Kiem and Li 2011] Y.-H. Kiem and J. Li, "Low degree GW invariants of spin surfaces", Pure Appl. Math. Q. 7:4 (2011), 1449-1475. MR 2918169 Zbl 06107784

[Lee 2013] J. Lee, "Sum formulas for local Gromov-Witten invariants of spin curves", Trans. Amer. Math. Soc. 365: 1 (2013), 459-490. MR 2984064

[Lee and Parker 2007] J. Lee and T. H. Parker, "A structure theorem for the Gromov-Witten invariants of Kähler surfaces”, J. Differential Geom. 77:3 (2007), 483-513. MR 2010b:53159 Zbl 1130.53059

[Lee and Parker 2012] J. Lee and T. H. Parker, "Recursion formulas for spin Hurwitz numbers", preprint, 2012. arXiv 1212.1825

[Maulik and Pandharipande 2008] D. Maulik and R. Pandharipande, "New calculations in GromovWitten theory", Pure Appl. Math. Q. 4:2 (2008), 469-500. MR 2009d:14073 Zbl 1156.14042

[Mumford 1971] D. Mumford, "Theta characteristics of an algebraic curve", Ann. Sci. École Norm. Sup. (4) 4 (1971), 181-192. MR 45 \#1918 Zbl 0216.05904

[Okounkov and Pandharipande 2006] A. Okounkov and R. Pandharipande, "Gromov-Witten theory, Hurwitz theory, and completed cycles”, Ann. of Math. (2) 163:2 (2006), 517-560. MR 2007b:14123 Zbl 1105.14076 
Received May 7, 2012. Revised September 1, 2012.

JUNHO LEE

DEPARTMENT OF MATHEMATICS

UNIVERSITY OF CENTRAL FLORIDA

ORLANDO, FL 32816

UNITED STATES

junlee@mail.ucf.edu 


\title{
PACIFIC JOURNAL OF MATHEMATICS
}

\author{
msp.org/pjm
}

Founded in 1951 by E. F. Beckenbach (1906-1982) and F. Wolf (1904-1989)

\section{EDITORS}

V. S. Varadarajan (Managing Editor)

Department of Mathematics

University of California

Los Angeles, CA 90095-1555

pacific@math.ucla.edu

Paul Balmer

Department of Mathematics

University of California

Los Angeles, CA 90095-1555

balmer@math.ucla.edu

Daryl Cooper

Department of Mathematics

University of California

Santa Barbara, CA 93106-3080 cooper@math.ucsb.edu

Jiang-Hua $\mathrm{Lu}$

Department of Mathematics

The University of Hong Kong

Pokfulam Rd., Hong Kong jhlu@maths.hku.hk
Don Blasius

Department of Mathematics University of California

Los Angeles, CA 90095-1555

blasius@math.ucla.edu

Robert Finn

Department of Mathematics Stanford University

Stanford, CA 94305-2125

finn@math.stanford.edu

Sorin Popa

Department of Mathematics

University of California

Los Angeles, CA 90095-1555

popa@math.ucla.edu

Paul Yang

Department of Mathematics

Princeton University

Princeton NJ 08544-1000

yang@math.princeton.edu

\section{PRODUCTION}

Silvio Levy, Scientific Editor, production@msp.org

\section{SUPPORTING INSTITUTIONS}

ACADEMIA SINICA, TAIPEI

CALIFORNIA INST. OF TECHNOLOGY

INST. DE MATEMÁTICA PURA E APLICADA

KEIO UNIVERSITY

MATH. SCIENCES RESEARCH INSTITUTE

NEW MEXICO STATE UNIV.

OREGON STATE UNIV.

\author{
STANFORD UNIVERSITY \\ UNIV. OF BRITISH COLUMBIA \\ UNIV. OF CALIFORNIA, BERKELEY \\ UNIV. OF CALIFORNIA, DAVIS \\ UNIV. OF CALIFORNIA, LOS ANGELES \\ UNIV. OF CALIFORNIA, RIVERSIDE \\ UNIV. OF CALIFORNIA, SAN DIEGO \\ UNIV. OF CALIF., SANTA BARBARA
}

\author{
Vyjayanthi Chari \\ Department of Mathematics \\ University of California \\ Riverside, CA 92521-0135 \\ chari@math.ucr.edu \\ Kefeng Liu \\ Department of Mathematics \\ University of California \\ Los Angeles, CA 90095-1555 \\ liu@math.ucla.edu \\ Jie Qing \\ Department of Mathematics \\ University of California \\ Santa Cruz, CA 95064 \\ qing@cats.ucsc.edu
}

These supporting institutions contribute to the cost of publication of this Journal, but they are not owners or publishers and have no responsibility for its contents or policies.

See inside back cover or msp.org/pjm for submission instructions.

The subscription price for 2013 is US \$400/year for the electronic version, and \$485/year for print and electronic.

Subscriptions, requests for back issues and changes of subscribers address should be sent to Pacific Journal of Mathematics, P.O. Box 4163, Berkeley, CA 94704-0163, U.S.A. The Pacific Journal of Mathematics is indexed by Mathematical Reviews, Zentralblatt MATH, PASCAL CNRS Index, Referativnyi Zhurnal, Current Mathematical Publications and the Science Citation Index.

The Pacific Journal of Mathematics (ISSN 0030-8730) at the University of California, c/o Department of Mathematics, 798 Evans Hall \#3840, Berkeley, CA 94720-3840, is published monthly except July and August. Periodical rate postage paid at Berkeley, CA 94704, and additional mailing offices. POSTMASTER: send address changes to Pacific Journal of Mathematics, P.O. Box 4163, Berkeley, CA 94704-0163.

PJM peer review and production are managed by EditFLOW ${ }^{\circledR}$ from Mathematical Sciences Publishers.

PUBLISHED BY

mathematical sciences publishers

nonprofit scientific publishing

http://msp.org/

(C) 2013 Mathematical Sciences Publishers 


\section{PACIFIC JOURNAL OF MATHEMATICS}

Volume $263 \quad$ No. $2 \quad$ June 2013

Realizations of $B C_{r}$-graded intersection matrix algebras with grading 257 subalgebras of type $B_{r}, r \geq 3$

SANDEEP BHARGAVA and YUN GAO

Stable flags, trivializations and regular connections

283

Elie COMPOINT and EDUARDo COREL

Elliptic aliquot cycles of fixed length

353

NATHAN JONES

Asymptotic $L^{4}$ norm of polynomials derived from characters

DANIEL J. KATZ

Degree-three spin Hurwitz numbers

JUNHO LEE

$\left(\mathbb{Z}_{2}\right)^{3}$-colorings and right-angled hyperbolic 3-manifolds

YOULIN LI and JiMING MA

Real closed separation theorems and applications to group algebras

Tim NETZER and ANDREAS THOM

Uniqueness theorem for ordinary differential equations with Hölder continuity

YifEI PAN, MEI WANG and Yu YAN

An analogue to the Witt identity

G. A. T. F. DA CostA and G. A. ZimmermanN

On the classification of stable solutions to biharmonic problems in large dimensions

Juncheng Wei, Xingwang Xu and Wen Yang 